\title{
Telomere shortening is associated with poor physical performance in knee osteoarthritis
}

\author{
PACHAREE MANOY ${ }^{1}$, PONGSAK YUKTANANDANA $^{2}$, AREE TANAVALEE $^{2}$, \\ THANATHEP TANPOWPONG ${ }^{2}$, THAN ITTIPANICHPONG ${ }^{2}$ and SITTISAK HONSAWEK ${ }^{2,3}$
}

${ }^{1}$ Unit of Excellence for Physical Fitness and Exercise, Department of Physical Therapy, School of Allied Health Sciences, University of Phayao, Phayao 56000; ${ }^{2}$ Vinai Parkpian Orthopaedic Research Center, Department of Orthopaedics, Faculty of Medicine, Chulalongkorn University, King Chulalongkorn Memorial Hospital, Thai Red Cross Society;

${ }^{3}$ Department of Biochemistry, Osteoarthritis and Musculoskeleton Research Unit, Faculty of Medicine, Chulalongkorn University, King Chulalongkorn Memorial Hospital, Thai Red Cross Society, Bangkok 10330, Thailand

Received December 24, 2019; Accepted June 24, 2020

DOI: $10.3892 /$ br.2020.1334

\begin{abstract}
Telomere length is a hallmark characteristic of ageing and age-related diseases. Osteoarthritis (OA) is the most common cause of joint pain and physical disability in the elderly. Previous studies have revealed the role of telomere shortening in OA; however, the relationship between telomere length, muscle strength and physical performance in knee OA patients remains unknown. The aim of the present study was to investigate the association of telomere length and physical performance in patients with knee OA. A total of 202 patients with knee OA and 60 healthy controls were enrolled in the study. The quality of life was assessed using Western Ontario and McMaster Universities Osteoarthritis (WOMAC) index and Short Form Health Survey. The skeletal muscle mass was examined using bioelectrical impedance analysis, while the muscle strength was analyzed using hand grip force and isometric knee extension force. The physical performance of patients with knee OA was also investigated using gait speed,
\end{abstract}

Correspondence to: Professor Sittisak Honsawek, Department of Biochemistry, Osteoarthritis and Musculoskeleton Research Unit, Faculty of Medicine, Chulalongkorn University, King Chulalongkorn Memorial Hospital, Thai Red Cross Society, 1873 Rama IV Road Pathumwan, Bangkok 10330, Thailand

E-mail: sittisak.h@chula.ac.th

Abbreviations: BIA, bioelectrical impedance analysis; BMI, body mass index; Fat \%, percentage of total fat mas; FM, fat mass; MCS, mental health composite scores; 6MWT, 6-min walk test; OA, osteoarthritis; OR, odds ratio; PCS, physical health composite scores; RT-qPCR, reverse transcription-quantitative PCR; ROS, reactive oxygen species; RTL, relative telomere length; SF-12, Short Form Health Survey; SMI, skeletal muscle index; STS, sit to stand test; TUGT, timed up and go test; WC, waist circumference; WOMAC, Western Ontario and McMaster Universities Osteoarthritis Index

Key words: telomere length, knee, OA, physical performance
Timed up and go test (TUGT), Sit to stand test and 6-min walk test (6MWT). Blood leukocyte relative telomere length (RTL) was assessed using real time quantitative PCR. The mean blood leukocyte RTL in knee OA subjects was significantly lower compared with healthy controls $(\mathrm{P}<0.001)$. Knee OA patients with RTL values in the lowest quartile had a slow gait speed $(\mathrm{P}=0.006)$ and prolonged TUGT time $(\mathrm{P}=0.03)$. Multivariate regression analyses and multiple logistic regression analyses adjusted for age, sex, waist circumference, body mass index, fat mass, skeletal muscle index and the total WOMAC demonstrated that gait speed, TUGT and 6MWT were associated with longer RTL (P-trend<0.05). These findings suggested that poorer physical performance was associated with shorter RTL. Therefore, leukocyte telomere length and physical performance tests, especially gait speed, TUGT and 6MWT, could predict the health status and quality of life in patients with knee OA.

\section{Introduction}

Osteoarthritis (OA) is the most common joint disorder that results from a degradation of the articular cartilage, osteophyte formation and subchondral bone sclerosis (1). The symptoms of the disease include pain, decreased range of motion and physical function that leads to mobility impairment later in life (2). Knee OA remains a major cause of lower limb disability, particularly in the elderly, and has been recognized as a major global health problem. The prevalence of knee OA in people aged $>55$ years is $15.6 \%$ in male and $30.5 \%$ in female (3). Moreover, there are multiple factors that can affect OA pathogenesis such as aging, mechanical stress, low level inflammation, environmental factors and genetic factors (4).

Telomere length is a potential valuable biomarker for biological age and several age-related chronic degenerative diseases $(5,6)$. Telomeres are tandem repeats at the chromosomal ends, consisting of repetitive DNA sequences of TTAGGG, that serve a critical role in maintaining the integrity of the genome (6). The capping function of telomeres protects 
chromosomes from degrading and prevents the recombination and end-to-end fusion between chromosomes (6). When cells divide, the telomere shortens as DNA polymerases are not capable of completely replicating the chromosomes; this phenomenon was revealed to be the end-replication issue that causes the cells to enter into a state of senescence, apoptosis and inability to replicate $(6,7)$. Therefore, telomere length has been suggested as a biomarker for cellular aging and health status, and is associated with mortality $(5,8)$. In a previous cardiovascular health study, telomere length was revealed to be associated with physical activity and physical fitness $(9,10)$.

Previous studies have reported that OA is closely associated with telomere shortening in the blood and cartilage of patients with different forms of OA $(11,12)$. However, to the best of our knowledge, the relationships between telomere length, muscle strength and physical performance in patients with knee OA have not been previously investigated. Thus, it was hypothesized that telomere shortening may be associated with poor muscle strength and physical performance in patients with knee OA. Therefore, the present study investigated the relationship between leukocyte telomere length, muscle strength and physical performance in patients with knee OA.

\section{Materials and methods}

Ethics. The study protocol conformed to the ethical standards outlined in the Declaration of Helsinki and was approved by the Institutional Review Board on Human Research of the Faculty of Medicine, Chulalongkorn University. Patients that were eligible for the study were approached. Knee OA participants who met the criteria of American College of Rheumatology (13) and were aged 50-80 years were enrolled in the present study. Information pertaining to the study was provided to all patients, and written informed consent was obtained from all participants.

Study participants. A cross-sectional study was conducted in 202 patients with knee OA (181 females; 21 males; aged 50-80 years, mean age, 65.35 years). All procedures were performed at the outpatient clinic of the Department of Orthoapedics, King Chulalongkorn Memorial Hospital between January and December 2015. The exclusion criteria for knee OA participants included the Kellgren-Lawrence grading $>3$ (14), history of knee surgery and other forms of arthritis than OA (such as septic arthritis, rheumatoid arthritis and gout). In total, 60 healthy volunteers with no clinical or radiographic evidence of OA (34 females; 26 males; aged 50-80 years; mean age, 62.25 years) were enrolled in the current study.

Clinical parameter assessment. Knee OA participants completed a self-report pain questionnaire designed by the Western Ontario and McMaster Universities Osteoarthritis Index (WOMAC) (15) and health-related quality of life questionnaire (16). WOMAC was used to assess the pain (15), stiffness and physical disability of the patient, based on a scale that ranged between 0-10. A total WOMAC score was created by summing the items for all three subscales and a high score indicated worst pain, stiffness and physical disability (15). The Thai version of the Short Form Health Survey (SF-12) was used to evaluate the health-related quality of life, which included physical health composite scores and mental health composite scores (MCS) that ranged between 0-100; higher scores indicated improved health (16).

Anthropometric measurements and skeletal muscle index (SMI). The participant's height and weight were measured to calculate the participant's body mass index (BMI; weight in $\mathrm{kg} /$ height in $\mathrm{m}^{2}$ ). The waist circumference (WC) was also measured. Bioelectrical impedance analysis (BIA; Tanita BC-418; Tanita) was used to evaluate the percentage of total fat mass (Fat \%), fat mass (FM) and visceral fat rate. The SMI was obtained from the sum of the percentages of the skeletal muscle mass of the arms and legs, excluding the trunk, that were divided by the body weight $(\%)$.

Muscle strength. Grip strength of the dominant (preferred) and non-dominant (not preferred) hands was examined using a grip strength dynamometer (Takei Scientific Instruments Co., Ltd.). The force was recorded in $\mathrm{kg}$. The best of the three trials was presented as the maximal squeeze.

Quadriceps strength in symptomatic and non-symptomatic legs was assessed with a hand-held dynamometer (MicroFET 2; Hoggan). The force was recorded in Newtons (N). The participants were seated on the examination table and their knees were flexed to $90^{\circ}$. The lower legs were vertical to the floor and the quadriceps strength was assessed when the knees were flexed at $90^{\circ}$. The participants then raised their lower legs and held this position against the maximum persistent force $(5 \mathrm{sec})$ applied by the physical therapist via the hand-held dynamometer, which was pointed on the anterior part of the lower leg, $5-\mathrm{cm}$ above the proximal ankle and on a vertical line to the tibia crest. The mean of the three trials represented the maximum quadriceps strength.

Physical performance. To assess the usual $4 \mathrm{~m}$ gait speed, the participants were instructed to walk at a normal pace. The time to walk $4 \mathrm{~m}$ was recorded with a standard stopwatch and the gait speed was calculated as the walking distance divided by the time. The gait speed was measured twice, and the fastest time was used.

As for the timed up and go test (TUGT), the participants sat in a standard chair. Then, the participants were requested to stand up from the chair and walk a distance of $3 \mathrm{~m}$ at a comfortable pace, and then return to the chair to sit down again.

For the Sit to stand test (STS), the participants performed five consecutive chair stands from a standard chair that was 45-cm high as quickly as possible with their arms across their chest. This activity was measured in sec.

With regards to the 6-min Walk (6MWT) test, the participants were requested to cover as much distance as possible at a self-paced walking velocity within $6 \mathrm{~min}$ on a flat surface that was $25 \mathrm{~m}$ long.

Measurement of the telomere length. Genomic DNA was extracted from peripheral blood leukocytes ( $3 \mathrm{ml}$ whole blood) using a commercial DNA isolation kit (GF-1 blood DNA extraction kit; Vivantis Technologies Sdn Bhd). Leukocyte telomere length was measured as a ratio of the telomere repeat copy number (T) to the single-copy gene copy number $(\mathrm{S} ; \mathrm{T} / \mathrm{S}$ ratio) using quantitative real time PCR (qPCR) as described previously (17).

qPCR was performed using a StepOnePlus Real Time PCR system (Applied Biosystems; Thermo Fisher Scientific, Inc.) 
Table I. Baseline characteristics of 202 patients with knee OA.

\begin{tabular}{|c|c|c|c|}
\hline Characteristics & Patients with knee OA & Healthy controls & P-value \\
\hline Age, years & $65.35 \pm 5.1$ & $62.25 \pm 6.42$ & 0.5 \\
\hline Sex & & & 0.4 \\
\hline Female & 181 & 34 & \\
\hline Male & 21 & 26 & \\
\hline RTL, T/S ratio & $0.59 \pm 0.10$ & $1.31 \pm 0.30$ & $<0.001$ \\
\hline BMI, $\mathrm{kg} / \mathrm{m}^{2}$ & $25.50 \pm 0.29$ & $25.55 \pm 1.21$ & 0.6 \\
\hline Waist circumference, $\mathrm{cm}$ & $87.99 \pm 0.70$ & NA & NA \\
\hline \multicolumn{4}{|l|}{ Body composition } \\
\hline Fat $\%$ & $35.22 \pm 0.52$ & NA & NA \\
\hline Fat mass, kg & $22.59 \pm 0.60$ & NA & NA \\
\hline Visceral fat rating, $\%$ & $9.70 \pm 0.29$ & NA & NA \\
\hline SMI, $\%$ & $28.55 \pm 0.28$ & NA & NA \\
\hline \multicolumn{4}{|l|}{ WOMAC } \\
\hline Pain, 0-10 & $2.64 \pm 0.14$ & NA & NA \\
\hline Stiffness, $0-10$ & $2.75 \pm 0.17$ & NA & NA \\
\hline Physical disability, 0-10 & $3.08 \pm 0.15$ & NA & NA \\
\hline Total score, $0-10$ & $2.80 \pm 0.13$ & NA & NA \\
\hline \multicolumn{4}{|l|}{ SF-12 } \\
\hline PCS, $0-100$ & $38.04 \pm 0.65$ & NA & NA \\
\hline MCS, $0-100$ & $49.03 \pm 0.65$ & NA & NA \\
\hline
\end{tabular}

Data are presented as the mean \pm SEM. BMI, Body mass index; RLT, Relative telomere length; NA, Not available; T/S ratio, ratio of telomere repeat copy number to single gene copy number; SMI, Skeletal muscle index; WOMAC, Western Ontario and McMaster Universities Osteoarthritis Index; SF-12, Short form health survey; PCS, Physical health composite scores; MCS, Mental health composite scores; Fat \%, Percentage of total fat mass; OA, osteoarthritis.

with SYBR-Green fluorescence (RBC Bioscience). Briefly, two pairs of primers were used to amplify the telomere repeats copy number relative to another 36B4 for the amplification of the single-copy nuclear gene. The primers utilized for telomere repeat copy number were as follows: Telomere forward, 5'-CGG TTTGTTTGGGTTTGGGTTTGGGTTTGGGTTTGGGTT-3' and reverse, 5'-GGCTTGCCTTACCCTTACCCTTACCCT TACCCTTACCCT-3'. For the single-copy gene, the following primers were used: 36B4 forward, 5'-CAGCAAGTGGGAAGG TGTAATCC-3' and reverse, 5'-CCCATTCTATCATCAACG GGTACAA-3'. The thermocycling conditions for the telomeres and single copy genes included: Initial denaturation at $95^{\circ} \mathrm{C}$ for $10 \mathrm{~min}$, followed by 40 cycles of $15 \mathrm{sec}$ at $95^{\circ} \mathrm{C}$ and $1 \mathrm{~min}$ at $54^{\circ} \mathrm{C}$. All samples were performed in duplicate for both telomere and 36B4 reactions in the same run using 2 ng DNA per $10 \mu \mathrm{l}$ reaction. The relative $\mathrm{T} / \mathrm{S}$ ratio was calculated by using the $2^{-\Delta \Delta \mathrm{Cq}}$ method $(18,19)$.

Statistical analysis. Experiments were performed in triplicate and repeated at least twice. Statistical analyses were performed using the SPSS software v.22.0 for Windows (IBM Corp.). Statistical significance between baseline variables of the healthy controls and OA participants was determined using $\chi^{2}$ test and unpaired Student's t-test. Comparisons between the means of the quartile of RTL were performed using one-way ANOVA with Tukey's post hoc test if ANOVA showed significance.
Correlations were analyzed using Spearman's rank correlation and multivariate linear regression analysis. Multiple logistic regression analysis was performed to determine the strength of association between RTL, muscle strength and physical performance using the age, sex, WC, BMI, FM, SMI and total of WOMAC as the covariates and odds ratio (OR) with a $95 \% \mathrm{CI}$. Data are presented as the mean \pm SEM. $\mathrm{P}<0.05$ was considered to indicate a statistically significant difference.

\section{Results}

Participant characteristics. A total of 202 patients with knee $\mathrm{OA}$ and 60 healthy controls were age-matched $(\mathrm{P}=0.5)$ and sex-matched $(\mathrm{P}=0.4)$, and the baseline characteristics are presented in Table I. The female:male ratio was 181:21 for knee OA participants and 34:26 for the controls $(\mathrm{P}=0.4)$. The mean blood leukocyte RTL in knee OA subjects was significantly lower compared with the healthy controls $(0.59 \pm 0.10$ vs. $1.31 \pm 0.30$; $\mathrm{P}<0.001)$. Moreover, there was no significant difference in the BMI between the OA participants and the controls $(\mathrm{P}>0.05)$.

The OA participants were subsequently classified into the good outcome group $(n=135)$ and the poor outcome group $(\mathrm{n}=67)$, as previously described by Colbert et al (20). There was no statistically significant difference in the mean leukocyte RTL between knee OA participants with good and poor outcomes $(0.59 \pm 0.02$ vs. $0.58 \pm 0.02 ; \mathrm{P}=0.948)$. 
Table II. Comparison of the characteristics between female and male patients with knee OA.

Patients with knee OA

\begin{tabular}{|c|c|c|c|}
\hline \multirow[b]{2}{*}{ Variables } & & \multirow[b]{2}{*}{ P-value } \\
\hline & Females $(\mathrm{n}=181)$ & Males $(n=21)$ & \\
\hline Age, years & $65.32 \pm 0.53$ & $65.71 \pm 1.86$ & 0.81 \\
\hline RTL, T/S ratio & $0.58 \pm 0.01$ & $0.59 \pm 0.05$ & 0.86 \\
\hline $\mathrm{BMI}, \mathrm{kg} / \mathrm{m}^{2}$ & $25.17 \pm 0.29$ & $28.31 \pm 1.18$ & 0.001 \\
\hline Waist circumference, $\mathrm{cm}$ & $86.79 \pm 0.68$ & $98.33 \pm 2.58$ & $<0.001$ \\
\hline \multicolumn{4}{|l|}{ Body composition } \\
\hline Fat $\%$ & $36.14 \pm 0.51$ & $27.52 \pm 1.75$ & $<0.001$ \\
\hline Fat mass, $\mathrm{kg}$ & $27.66 \pm 0.62$ & $22.11 \pm 2.25$ & 0.04 \\
\hline Visceral fat rating, $\%$ & $8.91 \pm 0.24$ & $16.28 \pm 1.09$ & $<0.001$ \\
\hline SMI, \% & $27.78 \pm 0.23$ & $35.02 \pm 0.97$ & $<0.001$ \\
\hline \multicolumn{4}{|l|}{ WOMAC } \\
\hline Pain, 0-10 & $2.67 \pm 0.15$ & $2.09 \pm 0.38$ & 0.20 \\
\hline Stiffness, 0-10 & $2.75 \pm 0.18$ & $2.48 \pm 0.49$ & 0.64 \\
\hline Physical disability, 0-10 & $3.11 \pm 0.15$ & $2.51 \pm 0.45$ & 0.21 \\
\hline Total score, $0-10$ & $2.81 \pm 0.14$ & $2.36 \pm 0.40$ & 0.29 \\
\hline \multicolumn{4}{|l|}{ SF-12 } \\
\hline PCS, 0-100 & $37.83 \pm 0.70$ & $40.40 \pm 1.81$ & 0.22 \\
\hline MCS, 0-100 & $48.98 \pm 0.71$ & $49.82 \pm 1.60$ & 0.69 \\
\hline \multicolumn{4}{|l|}{ Muscle strength } \\
\hline \multicolumn{4}{|l|}{ Grip strength, kg } \\
\hline Dominant & $21.03 \pm 0.30$ & $32.72 \pm 1.45$ & $<0.001$ \\
\hline Non-dominant & $19.02 \pm 0.30$ & $30.52 \pm 1.39$ & $<0.001$ \\
\hline \multicolumn{4}{|l|}{ Knee extension force, $\mathrm{N}$} \\
\hline Symptomatic leg & $365.49 \pm 7.16$ & $432.75 \pm 20.83$ & 0.003 \\
\hline Non-symptomatic leg & $417.31 \pm 7.71$ & $478.15 \pm 20.43$ & 0.01 \\
\hline \multicolumn{4}{|l|}{ Physical performance } \\
\hline Gait speed, m/sec & $0.93 \pm 0.01$ & $1.01 \pm 0.04$ & 0.09 \\
\hline TUGT, sec & $9.96 \pm 0.19$ & $9.46 \pm 0.48$ & 0.40 \\
\hline STS, sec & $15.21 \pm 0.35$ & $12.64 \pm 0.611$ & 0.01 \\
\hline 6MWT, m & $365.84 \pm 6.09$ & $382.90 \pm 21.38$ & 0.38 \\
\hline
\end{tabular}

Data are presented as the mean \pm SEM. Each experiment was performed in duplicate. BMI, Body mass index; RLT, Relative telomere length; NA, Not available; T/S ratio, ratio of telomere repeat copy number to single gene copy number; Fat \%, Percentage of total fat mass; SMI, Skeletal muscle index; WOMAC, Western Ontario and McMaster Universities Osteoarthritis Index; SF-12, Short form health survey; PCS, Physical health composite scores; MCS, Mental health composite scores; OA, osteoarthritis; N, Newton.

Comparison between female and male knee OA participants. In the stratified analysis, according to sex (Table II), there were no significant differences in age and the mean leukocyte RTL, WOMAC, SF-12 and physical performance between female and male participants $(\mathrm{P}>0.05)$. However, male participants had significantly higher BMI, WC, visceral fat rating, SMI and muscle strength compared with the female participants $(\mathrm{P}<0.05)$. In contrast, Fat \% and FM were significantly greater in females compared with male participants $(\mathrm{P}<0.05)$.

Quartile of blood leukocyte RTL of knee OA participants. The RTL values were further separated into quartile levels. Participants with RTL values in the 1st quartile had the shortest telomere length. If the RTL values were in the 4 th quartile, then it indicated that the telomere length was the longest. Table III presents the characteristics of the participants in each group. There were no significant differences between participants with short telomere lengths (1st quartile) or long telomere lengths (4th quartile) for age, BMI, WC, body composition using BIA analysis, WOMAC and muscle strength $(\mathrm{P}>0.05)$. However, the participants with RTL values in the 1st quartile had the lowest MCS scores for SF-12, slowest gait speed and longer time for TUGT compared with the other quartiles.

Relationship of RTL, muscle strength and physical performance in patients with knee OA. There were no correlations between blood leukocyte RTL with grip strength (rho=0.04, $\mathrm{P}=0.53)$ and knee extension force $(\mathrm{rho}=0.02, \mathrm{P}=0.77)$. 
Table III. Demographic characteristics of patients with knee osteoarthritis according to the quartile of RTL.

\begin{tabular}{|c|c|c|c|c|c|}
\hline \multirow[b]{2}{*}{ Variables } & \multicolumn{4}{|c|}{ RTL } & \multirow[b]{2}{*}{ P-value } \\
\hline & 1st quartile $(\mathrm{n}=53)$ & 2nd quartile $(n=51)$ & 3rd quartile $(\mathrm{n}=50)$ & 4th quartile $(\mathrm{n}=48)$ & \\
\hline $\mathrm{RTL}, \mathrm{T} / \mathrm{S}$ ratio & $0.33 \pm 0.01$ & $0.49 \pm 0.01$ & $0.64 \pm 0.01$ & $0.90 \pm 0.02$ & $<0.001$ \\
\hline Age, years & $66.62 \pm 0.93$ & $65.24 \pm 0.87$ & $65.28 \pm 1.17$ & $64.13 \pm 1.11$ & 0.39 \\
\hline $\mathrm{BMI}, \mathrm{kg} / \mathrm{m}^{2}$ & $25.69 \pm 0.60$ & $25.38 \pm 0.48$ & $24.67 \pm 0.59$ & $26.27 \pm 0.69$ & 0.30 \\
\hline Waist circumference, $\mathrm{cm}$ & $89.14 \pm 1.44$ & $87.66 \pm 1.12$ & $85.86 \pm 1.53$ & $89.34 \pm 1.44$ & 0.28 \\
\hline \multicolumn{6}{|l|}{ Body composition } \\
\hline Fat $\%$ & $34.76 \pm 1.14$ & $36.17 \pm 0.87$ & $34.13 \pm 1.18$ & $35.88 \pm 0.97$ & 0.48 \\
\hline Fat mass, $\mathrm{kg}$ & $22.24 \pm 1.17$ & $23.13 \pm 1.03$ & $21.33 \pm 1.28$ & $23.75 \pm 1.34$ & 0.52 \\
\hline Visceral fat rating, $\%$ & $9.92 \pm 0.56$ & $9.26 \pm 0.45$ & $9.23 \pm 0.60$ & $10.41 \pm 0.70$ & 0.44 \\
\hline SMI, \% & $29.26 \pm 0.64$ & $27.67 \pm 0.46$ & $28.81 \pm 0.60$ & $28.44 \pm 0.52$ & 0.23 \\
\hline \multicolumn{6}{|l|}{ WOMAC } \\
\hline Pain, 0-10 & $2.48 \pm 0.33$ & $3.05 \pm 0.26$ & $2.33 \pm 0.26$ & $2.70 \pm 0.27$ & 0.31 \\
\hline Stiffness, 0-10 & $2.71 \pm 0.37$ & $2.93 \pm 0.34$ & $2.81 \pm 0.32$ & $2.54 \pm 0.37$ & 0.88 \\
\hline Physical disability, 0-10 & $3.32 \pm 0.33$ & $3.27 \pm 0.27$ & $2.84 \pm 0.26$ & $3.05 \pm 0.30$ & 0.38 \\
\hline Total score, $0-10$ & $2.84 \pm 0.30$ & $3.04 \pm 0.26$ & $2.55 \pm 0.23$ & $2.76 \pm 0.27$ & 0.64 \\
\hline \multicolumn{6}{|l|}{ SF-12 } \\
\hline PCS, 0-100 & $38.25 \pm 1.36$ & $35.42 \pm 1.16$ & $40.10 \pm 1.29$ & $38.42 \pm 1.35$ & 0.09 \\
\hline MCS, 0-100 & $46.30 \pm 1.05$ & $51.40 \pm 1.33$ & $48.96 \pm 1.47$ & $49.70 \pm 1.31$ & $0.04^{\mathrm{a}}$ \\
\hline \multicolumn{6}{|l|}{ Muscle strength } \\
\hline \multicolumn{6}{|l|}{ Grip strength, kg } \\
\hline Dominant & $22.17 \pm 0.90$ & $22.04 \pm 0.53$ & $22.00 \pm 0.75$ & $23.01 \pm 0.95$ & 0.69 \\
\hline Non-dominant & $19.96 \pm 0.84$ & $19.76 \pm 0.58$ & $19.99 \pm 0.77$ & $21.06 \pm 0.98$ & 0.67 \\
\hline \multicolumn{6}{|l|}{ Knee extension force, $\mathrm{N}$} \\
\hline Symptomatic leg & $361.03 \pm 16.32$ & $378.63 \pm 14.30$ & $363.78 \pm 12.79$ & $382.74 \pm 11.25$ & 0.61 \\
\hline Non-symptomatic leg & $412.51 \pm 16.28$ & $427.39 \pm 15.3$ & $421.97 \pm 14.75$ & $429.23 \pm 12.06$ & 0.85 \\
\hline \multicolumn{6}{|l|}{ Physical performance } \\
\hline Gait speed, m/sec & $0.87 \pm 0.02$ & $0.93 \pm 0.03$ & $0.99 \pm 0.02$ & $0.98 \pm 0.01$ & $0.006^{\mathrm{b}}$ \\
\hline TUGT, sec & $10.76 \pm 0.40$ & $9.82 \pm 0.36$ & $9.57 \pm 0.29$ & $9.42 \pm 0.29$ & $0.03^{\mathrm{b}}$ \\
\hline STS, sec & $15.58 \pm 0.68$ & $15.75 \pm 0.70$ & $14.67 \pm 0.57$ & $13.59 \pm 0.56$ & 0.07 \\
\hline 6MWT, m & $344.49 \pm 11.89$ & $370.60 \pm 12.03$ & $376.86 \pm 11.66$ & $380.73 \pm 10.47$ & 0.11 \\
\hline
\end{tabular}

${ }^{\mathrm{a}} \mathrm{P}<0.051$ st vs. 2nd quartile; ${ }^{\mathrm{b}} \mathrm{P}<0.05$ 1st vs. 3rd and 4th quartile, using ANOVA with post hoc analysis. Data are presented as the mean $\pm \mathrm{SEM}$. Each experiment was performed in duplicate. RTL, Relative telomere length; BMI, Body mass index; Fat \%, Percentage of total fat mass; SMI, Skeletal muscle index; WOMAC, Western Ontario and McMaster Universities Osteoarthritis Index; SF-12, 12-Item short form health survey; PCS, Physical health composite scores; MCS, Mental health composite scores; TUGT, Timed up and go test; STS, Sit to stand; 6MWT, 6-min walk test; N, Newton.

However, there was a weak correlation between blood leukocyte RTL and physical performance. Leukocyte RTL was also demonstrated to be weakly positively correlated (21) with gait speed (rho=0.20; $\mathrm{P}=0.004)$ and 6MWT (rho=0.21; $\mathrm{P}=0.003$ ). In addition, leukocyte RTL were negatively weakly correlated with TUTG (rho=-0.16; $\mathrm{P}=0.03)$ and STS (rho=-0.15; $\mathrm{P}=0.03$ ) (Fig. 1).

Multiple linear regression models of blood leukocyte RTL and physical performance. Subsequently, multivariate linear regression analysis of leukocyte RTL was performed, after adjustment for age, sex, WC, BMI, FM, skeletal muscle index and total of WOMAC, to estimate the interaction between RTL and physical performance (Table IV). Blood leukocyte RTL was positively associated with gait speed $(\beta$ coefficient $=0.185$; $\mathrm{P}=0.023)$ and 6MWT $(\beta$ coefficient $=0.191 ; \mathrm{P}=0.022)$. In contrast, RTL was found to be negatively associated with TUGT $(\beta$ coefficient $=-0.189 ; \mathrm{P}=0.025)$ and $\mathrm{STS}(\beta$ coefficient $=-0.231$; $\mathrm{P}=0.004)$.

Multiple logistic regression analysis between $R T L$, muscle strength and physical performance. The multiple logistic regression models were used to estimate the impact of muscle strength and physical performance on RTL based on the potential influential covariates (Table V). After adjusting for age and sex, a significant effect of physical performance on 
A

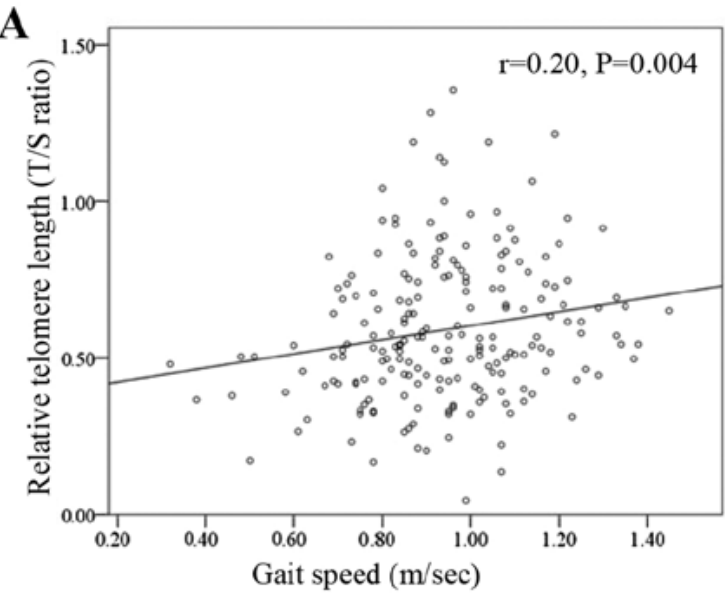

C

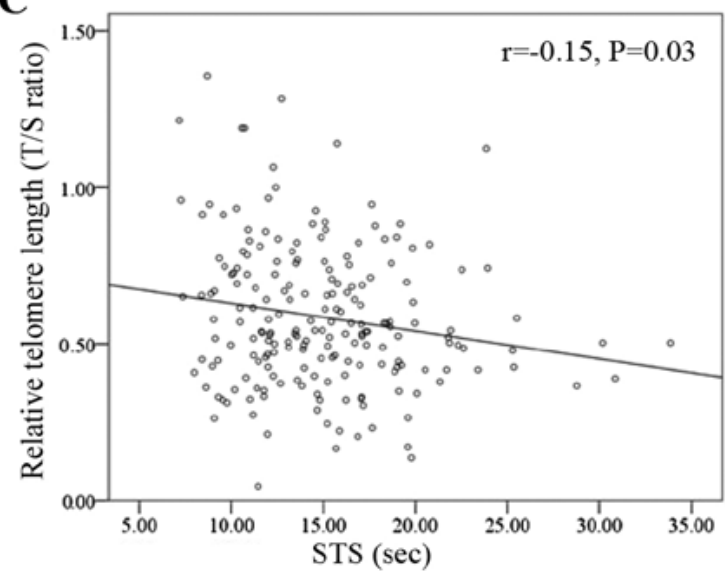

B

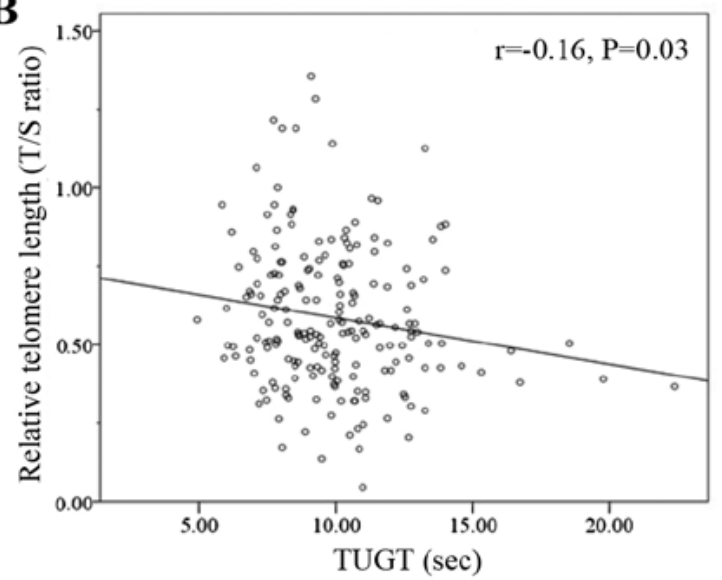

D

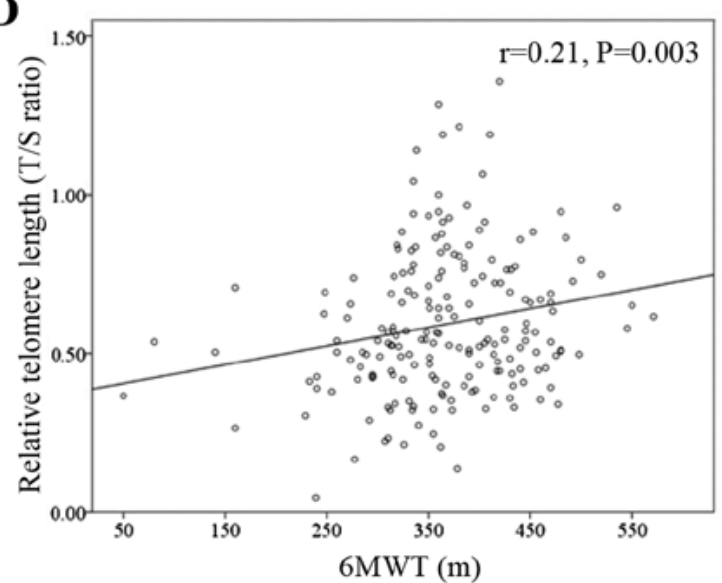

Figure 1. Scatter diagram and correlation analysis of blood leukocyte relative telomere length and physical performance tests in patients with knee osteoarthritis. Relative telomere length was directly associated with (A) gait speed. Relative telomere length was inversely associated with (B) TUGT and (C) STS . Relative telomere length was directly associated with (D) 6MWT. TUGT, timed up and go test; STS, Sit to stand; 6MWT, 6-min walk test; T/S, ratio of telomere repeat copy number to single gene copy number.

Table IV. Multiple linear regression analysis of RTL, muscle strength and physical performance.

Multivariate adjusted $^{\mathrm{a}}$

Variables

$\beta$ coefficients $(95 \% \mathrm{CI}) \quad \mathrm{P}$-value

Muscle strength

Grip strength, $\mathrm{kg}$

$0.032(-0.007-0.009) \quad 0.752$

Knee extension force, $\mathrm{N}$

$0.004(-0.008-0.008)$

0.948

Physical performance

$\begin{array}{lcc}\text { Gait speed, m/sec } & 0.185(0.031-0.407) & 0.023 \\ \text { TUGT, sec } & -0.189(-0.032-0.002) & 0.025 \\ \text { STS, sec } & -0.231(-0.019-0.004) & 0.004 \\ \text { 6MWT, m } & 0.191(0.000-0.001) & 0.022\end{array}$

${ }^{a}$ Adjusted for age, sex, waist circumference, body mass index, fat mass, skeletal muscle index and total Western Ontario and McMaster Universities Osteoarthritis Index. N, Newton; TUGT, Timed up and go test; STS, Sit to stand; 6MWT, 6-min walk test.

RTL was identified; gait speed and 6MWT were significant predictors of RTL (P-trend<0.05). Multivariate-adjusted models controlled for age, sex, WC, BMI, FM, SMI and the total WOMAC demonstrated that gait speed, TUGT and $6 \mathrm{MWT}$ were associated with longer RTL (P-trend<0.05).

\section{Discussion}

The current cross-sectional study investigated the potential relationship between RTL, muscle strength and physical performance in patients with knee OA to determine whether physical disability may impact telomere length. It was found that patients with OA in the 1st quartile of telomere length had lower MCS scores for SF-12, slower gait speed and a longer time in the TUGT. Furthermore, from the multivariate linear regression model, gait speed, TUGT and 6MWT were associated with longer RTL. Therefore, the present results support the hypothesis that shorter RTL was associated with physical performance. However, the current data do not support the hypothesis that telomere length was associated with muscle strength. To the best of our knowledge, this study was the first to demonstrate the relationship between telomere length and physical performance in patients with knee OA.

Telomere shortening is a natural process of the somatic cells (22). Aging has been shown to accelerate the shortening of the telomeres, which can be observed in several age-related diseases, such as diabetes, cancer and diseases of the immune 
Table V. Multivariate logistic regression examining the odds ratio of upper and lower quartile of RTL based on the muscle strength and physical performance ${ }^{\mathrm{a}}$.

\begin{tabular}{|c|c|c|}
\hline Variables & $\begin{array}{l}\text { Age and sex adjusted } \\
\text { OR }(95 \% \mathrm{CI})\end{array}$ & $\begin{array}{c}\text { Multivariate adjusted } \\
\text { OR }(95 \% \mathrm{CI})\end{array}$ \\
\hline \multicolumn{3}{|c|}{ Grip strength, kg } \\
\hline$<18.60^{\mathrm{c}}$ & 1 & 1 \\
\hline $18.60-21.55$ & $1.74(0.52-5.78)$ & $1.30(0.36-4.60)$ \\
\hline $21.56-24.55$ & $0.90(0.28-2.86)$ & $0.89(0.26-3.04)$ \\
\hline$>24.55$ & $1.4(0.37-5.26)$ & $1.13(0.27-4.72)$ \\
\hline P-trend & 0.24 & 0.22 \\
\hline \multicolumn{3}{|c|}{ Knee extension force, $\mathrm{N}$} \\
\hline$<344^{\mathrm{c}}$ & 1 & 1 \\
\hline $344-421.25$ & $1.07(0.32-3.53)$ & $1.16(0.33-4.01)$ \\
\hline $421.26-485.5$ & $1.52(0.48-4.83)$ & $1.70(0.48-6.06)$ \\
\hline$>485.5$ & $0.77(0.24-2.51)$ & $0.95(0.28-3.24)$ \\
\hline P-trend & 0.14 & 0.24 \\
\hline \multicolumn{3}{|c|}{ Gait speed, m/sec } \\
\hline$<0.83^{\mathrm{c}}$ & 1 & 1 \\
\hline $0.83-0.94$ & $5.31(1.47-19.19)$ & $5.27(1.33-20.82)$ \\
\hline $0.95-1.07$ & $4.79(1.33-17.16)$ & $4.88(1.29-18.38)$ \\
\hline$>1.07$ & $5.06(1.35-0.18 .87)$ & $5.36(1.32-21.75)$ \\
\hline P-trend & 0.04 & $<0.05$ \\
\hline \multicolumn{3}{|c|}{ Timed up and go test, sec } \\
\hline$>10.63^{c}$ & 1 & 1 \\
\hline $9.72-10.62$ & $2.33(0.69-7.81)$ & $3.99(1.01-15.73)$ \\
\hline $8.04-9.71$ & $1.57(0.50-4.93)$ & $2.10(0.59-7.43)$ \\
\hline$<8.04$ & $3.68(1.18-11.49)$ & $6.07(1.64-22.48)$ \\
\hline P-trend & 0.14 & 0.04 \\
\hline \multicolumn{3}{|l|}{ Sit to Stand, sec } \\
\hline$>17.35^{\mathrm{c}}$ & 1 & 1 \\
\hline $14.62-17.35$ & $1.02(0.30-3.43)$ & $1.79(0.44-7.23)$ \\
\hline $11.59-14.61$ & $2.96(0.08-9.94)$ & $5.20(1.25-21.65)$ \\
\hline$<11.59$ & $2.43(0.78-7.59)$ & $4.07(1.00-16.48)$ \\
\hline P-trend & 0.16 & 0.08 \\
\hline \multicolumn{3}{|c|}{6 -min walk test, $\mathrm{m}$} \\
\hline$<320^{c}$ & 1 & 1 \\
\hline $320-364$ & $9.32(2.22-39.09)$ & $12.05(2.37-61.08)$ \\
\hline $365-425$ & $12.20(2.30-45.14)$ & $17.85(3.39-95.68)$ \\
\hline$>425$ & $7.47(1.60-34.71)$ & $11.11(2.01-61.31)$ \\
\hline P-trend & 0.01 & 0.007 \\
\hline
\end{tabular}

${ }^{\mathrm{a}}$ Muscle strength and physical performance score is an ordinal score based on quartiles. ${ }^{\mathrm{b}}$ Multivariate adjusted models controlled for age, sex, waist circumference, body mass index, fat mass, skeletal muscle index and total of Western Ontario and McMaster Universities Osteoarthritis Index. ${ }^{c}$ Reference value against which other values were respectively normalized to. $n=101$. OR, Odds ratio; N, Newton.

system (6). In addition, multiple factors such as age, obesity, genetic and environmental factors can cause OA (23). Previous studies have reported that OA is closely related to shorter telomere length (24). For instance, McAlindon et al (12) measured the leucocyte telomere length in patients with symptomatic hand $\mathrm{OA}$, and revealed that there were strong associations in the telomere length of leukocytes with the incident of radiographic $\mathrm{OA}$ in the interphalangeal joints of the hands.
Moreover, Tamayo et al (11) analyzed the telomere length of human chondrocytes and peripheral blood leukocytes. In patients with OA, the telomere length in chondrocytes was 1.6 times longer compared with that in leukocytes, whereas telomere length in chondrocytes was shorter compared with healthy volunteers (11). It was also shown that the frequency of numerical chromosomal abnormalities from leukocytes in patients with OA was higher compared with the healthy 
volunteers. Previous studies have revealed that there are no differences in the telomere length in peripheral blood leukocytes between patients with OA and healthy volunteers $(11,25)$. However, in the current study, blood leukocyte RTL in knee OA participants was significantly lower than that in the healthy controls. These findings support the hypothesis that patients with OA had shorter RTL compared with controls, and were in line with previous reports that the shortening of telomere length could be associated with age-related diseases $(5,6)$.

The current study identified a relationship between telomere length and physical performance in patients with knee OA, which was in line with the results reported by Soares-Miranda et al (9) who investigated the association between physical activity, physical performance and telomere length in older adults; a decline in the walking distance and a long time chair test were found to be associated with shorter telomeres. Furthermore, Lee et al (26) identified that elderly women with declining gait speed had shorter telomeres in their leukocytes. It has also been shown that slow gait speed, physical inactivity or a sedentary lifestyle can affect the length of the telomere, and its shortening is accelerated via the aging process that promotes cellular senescence $(27,28)$.

The present results indicated that the telomere length was not associated with muscle mass and muscle strength, such as grip strength and knee extension force. These findings support results from previous studies, which revealed that telomere length was not associated with handgrip strength and physical health (29-31). However, Woo et al (32) reported that longer telomere length was associated with slower decline in grip strength in community-dwelling older individuals. It has also been shown that there are no correlations between telomere length, sarcopenia, muscle mass, walking speed and chair stand test (32). In contrast, Marzetti et al (33) reported that telomere length was only related to muscle mass; short telomeres were observed in outpatients with sarcopenic geriatric. These conflicting findings indicate that additional studies are warranted to clarify the relationship between the telomere length, muscle mass, muscle strength, handgrip strength, sarcopenia, walking speed and chair stand test.

The precise mechanism of short telomeres in OA remains unknown. However, it has been suggested that the progressive degeneration of OA may be closely associated with oxidative stress and chronic inflammation (24). Reactive oxygen species (ROS) causes DNA damage when there are high levels of ROS, which occurs when there are single strand breaks (24), and accumulated levels of ROS may accelerate telomere shortening. Telomeric DNA sequences, TTAGGG repeats, are particularly sensitive to base oxidation due to the large number of guanine nucleotides that are readily oxidatively modified to 8 -oxyguanosine $(34,35)$. In human chondrocytes, ROS have been shown to induce telomere instability and result in replicative senescence and dysfunction $(36,37)$. Obesity and excessive mechanical loading on joints or malalignment can increase the production of oxidative stress, which can decrease the function of chondrocytes and induce chondrocyte senescence $(38,39)$. In addition, environmental and lifestyle factors may accelerate telomere shortening. For example, low levels of physical activity are associated with shorter telomere length (9), and the benefits of regular physical activity can upregulate antioxidant systems and reduce chronic systemic inflammation (9).
Accordingly, the balance between factors that promote oxidative stress and antioxidative defense may potentially influence the length of the telomeres. Moreover, the current results support the hypothesis that physical performance is significantly associated with blood leukocyte telomere length.

The present study has some limitations. First, this was a cross-sectional study, and the determination of direct cause and effect relationships could not be established. In addition, the timing of blood collection varied with respect to time since diagnosis and treatment, which introduces uncertainty regarding associations between telomere length and physical performance. Therefore, the associations identified in blood leukocyte DNA may indicate either casual, consequential or coincidental relationships. Thus, further cohort investigations are required to validate the cause-effect of telomere length on the changes in physical performance parameters. Second, the sample size was relatively small; this limits the statistical power of the current findings. Moreover, it was not possible to collect physical performance data in the controls. The subjects who participated in this study were specific to patients with $\mathrm{OA}$, in which elderly women have a high prevalence of knee OA (3), and thus it is challenging to compare results across studies, especially in male OA participants or in non-OA individuals. Third, several variables including age, genetic, physical exercise and/or lifestyle behaviors can influence the findings of the study. Due to the attempt to match the age between OA participants and the controls, the age range of the individuals enrolled into this study was narrow. As a result of this, the association between age and blood leukocyte RTL cannot be determined. Therefore, additional prospective longitudinal studies with a larger sample size are necessary to investigate the association between telomere shortening and the physical performance of participants with knee OA.

In conclusion, blood leukocyte RTL in patients with knee OA was significantly lower than that in the healthy controls. Moreover, shorter leukocyte RTL was associated with slow gait speed, a long time TUGT and 6MWT in these patients. The current results suggested that telomere shortening could be related to physical disability in participants with knee OA. These findings also supported the hypothesis that blood leukocyte telomere length may be a non-invasive biomarker that could provide information relating to the physical performance of the patient, and could predict physical activity in patients with knee OA.

\section{Acknowledgements}

The authors are grateful to Ms. Borwarnluck Thongtha, Mr. Surasit Suwannasin and Ms. Nungruthai Nilsri (Faculty of Medicine, Chulalongkorn University) for their technical assistance. The authors would also like to thank Ms. June Ohata and Dr Thananya Thongtan (Faculty of Medicine, Chulalongkorn University) for reviewing and proof-reading the manuscript.

\section{Funding}

The present was supported by the Osteoarthritis and Musculo skeleton Research Unit, 90th Anniversary Chulalongkorn University Fund and the Ratchadapiseksompotch Fund, Chulalongkorn University: CU_GR_63_95_30_02. 


\section{Availability of data and materials}

The datasets used and/or analyzed during the current study are available from the corresponding author on reasonable request.

\section{Authors' contributions}

PM, PY, AT, TT, TI and SH conceived and designed the experiments as well as performed the experiments and analyzed the data. PM and SH provided the reagents, materials and tools for analysis. PY, AT, TT, TI and SH enrolled the participants, obtained the written informed consents and collected the clinical data. PM and SH wrote and revised the manuscript. All authors have reviewed and approved the final version of this manuscript.

\section{Ethics approval and consent to participate}

The study protocol was approved by the Institutional Review Board on Human Research of the Faculty of Medicine, Chulalongkorn University. Information regarding the study was provided to the patients who were eligible to participate in the study. Written informed consent was obtained from the participants.

\section{Patient consent for publication}

Not applicable.

\section{Competing interests}

The authors declare that they have no competing interests.

\section{References}

1. Manoy P, Anomasiri W, Yuktanandana P, Tanavalee A, Mabey T and Honsawek S: Relationship of serum leptin and 25-hydroxyvitamin D in knee osteoarthritis patients. Chula Med J 62: 1037-1047, 2018.

2. Zhan D and Honsawek S: Reduction of leukocyte mitochondrial DNA copy number in knee osteoarthritis. Chula Med J 63: 207-209, 2019.

3. Litwic A, Edwards MH, Dennison EM and Cooper C: Epidemiology and burden of osteoarthritis. Br Med Bull 105 185-199, 2013.

4. Kuszel L, Trzeciak T, Richter M and Czarny-Ratajczak M: Osteoarthritis and telomere shortening. J Appl Genet 56: 169-176, 2015

5. Mather KA, Jorm AF, Parslow RA and Christensen H: Is telomere length a biomarker of aging? A review. J Gerontol A Biol Sci Med Sci 66: 202-213, 2011.

6. Xi H, Li C, Ren F, Zhang H and Zhang L: Telomere, aging and age-related diseases. Aging Clin Exp Res 25: 139-146, 2013.

7. Li D, Yuan Q and Wang W: The role of telomeres in musculoskeletal diseases. J Int Med Res 40: 1242-1250, 2012.

8. Fossel M: Use of telomere length as a biomarker for aging and age-related disease. Curr Transl Geriatrics Exp Gerontology Rep 1: 121-127, 2012.

9. Soares-Miranda L, Imamura F, Siscovick D, Jenny NS, Fitzpatrick AL and Mozaffarian D: Physical activity, physical fitness, and leukocyte telomere length: The cardiovascular health study. Med Sci Sports Exerc 47: 2525-2534, 2015.

10. Loprinzi PD: Cardiorespiratory capacity and leukocyte telomere length among adults in the United States. Am J Epidemiol 182: $198-201,2015$
11. Tamayo M, Mosquera A, Rego I, Blanco FJ, Gosálvez J and Fernández JL: Decreased length of telomeric DNA sequences and increased numerical chromosome aberrations in human osteoarthritic chondrocytes. Mutat Res 708: 50-58, 2011.

12. McAlindon T, Roberts M, Driban J, Schaefer L, Haugen IK, Smith SE, Duryea J, Cunha D, Blanco F, Fernández-Garcia JL and Eaton C: Incident hand OA is strongly associated with reduced peripheral blood leukocyte telomere length. Osteoarthritis Cartilage 26: 1651-1657, 2018.

13. Altman R, Asch E, Bloch D, Bole G, Borenstein D, Brandt K, Christy W, Cooke TD, Greenwald R and Hochberg M: Development of criteria for the classification and reporting of osteoarthritis. classification of osteoarthritis of the knee. Diagnostic and therapeutic criteria committee of the American rheumatism association. Arthritis Rheum 29: 1039-1049, 1986.

14. Spector TD and Cooper C: Radiographic assessment of osteoarthritis in population studies: Whither kellgren and lawrence? Osteoarthritis Cartilage 1: 203-206, 1993.

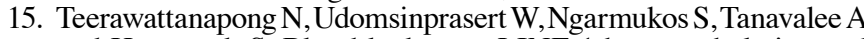
and Honsawek S: Blood leukocyte LINE-1 hypomethylation and oxidative stress in knee osteoarthritis. Heliyon 5: e01774, 2019.

16. Sakthong P, Kasemsup V and Winit-Watjana W: Assessment of health-related quality of life in thai patients after heart surgery. Asian Biomed 9: 203-210, 2015.

17. Udomsinprasert W, Poovorawan Y, Chongsrisawat V, Vejchapipat P, Zhan D and Honsawek S: Telomere length in peripheral blood leukocytes is associated with severity of biliary atresia. PLoS One 10: e0134689, 2015.

18. Livak KJ and Schmittgen TD: Analysis of relative gene expression data using real-time quantitative PCR and the 2(-Delta Delta C(T)) method. Methods 25: 402-408, 2001.

19. Poonpet T, Saetan N, Tanavalee A, Wilairatana V, Yuktanandana P and Honsawek S: Association between leukocyte telomere length and angiogenic cytokines in knee osteoarthritis. Int J Rheum Dis 21: $118-125,2018$

20. Colbert CJ, Song J, Dunlop D, Chmiel JS, Hayes KW, Cahue S, Moisio KC, Chang AH and Sharma L: Knee confidence as it relates to physical function outcome in persons with or at high risk of knee osteoarthritis in the osteoarthritis initiative. Arthritis Rheum 64: 1437-1446, 2012.

21. Schober P, Boer C and Schwarte LA: Correlation coefficients: Appropriate use and interpretation. Anesth Analg 126: 1763-1768, 2018.

22. Shammas MA: Telomeres, lifestyle, cancer, and aging. Curr Opin Clin Nutr Metab Care 14: 28-34, 2011.

23. Ding C, Jones G, Wluka AE and Cicuttini F: What can we learn about osteoarthritis by studying a healthy person against a person with early onset of disease? Curr Opin Rheumatol 22: 520-527, 2010

24. Fragkiadaki P, Nikitovic D, Kalliantasi K, Sarandi E, Thanasoula M, Stivaktakis PD, Nepka C, Spandidos DA, Tosounidis $\mathrm{T}$ and Tsatsakis A: Telomere length and telomerase activity in osteoporosis and osteoarthritis. Exp Ther Med 19: 1626-1632, 2020

25. Tamayo M, Mosquera A, Rego JI, Fernández-Sueiro JL, Blanco FJ and Fernandez JL: Differing patterns of peripheral blood leukocyte telomere length in rheumatologic diseases. Mutat Res 683: 68-73, 2010.

26. Lee JY, Bang HW, Ko JH, Kim JH and Lee DC: Leukocyte telomere length is independently associated with gait speed in elderly women. Maturitas 75: 165-169, 2013.

27. Sassenroth D, Meyer A, Salewsky B, Kroh M, Norman K, Steinhagen-Thiessen E and Demuth I: Sports and exercise at different ages and leukocyte telomere length in later life-data from the Berlin aging study II (BASE-II). PLoS One 10: e0142131, 2015.

28. Cherkas LF, Hunkin JL, Kato BS, Richards JB, Gardner JP, Surdulescu GL, Kimura M, Lu X, Spector TD and Aviv A: The association between physical activity in leisure time and leukocyte telomere length. Arch Intern Med 168: 154-158, 2008.

29. Bekaert S, Van Pottelbergh I, De Meyer T, Zmierczak H, Kaufman JM, Van Oostveldt P, and Goemaere S: Telomere length versus hormonal and bone mineral status in healthy elderly men. Mech Ageing Dev 126: 1115-1122, 2005.

30. Harris SE, Deary IJ, MacIntyre A, Lamb KJ, Radhakrishnan K, Starr JM, Whalley LJ and Shiels PG: The association between telomere length, physical health, cognitive ageing, and mortality in non-demented older people. Neurosci Lett 406: 260-264, 2006.

31. Mather KA, Jorm AF, Milburn PJ, Tan X, Easteal S and Christensen $\mathrm{H}$ : No associations between telomere length and age-sensitive indicators of physical function in mid and later life. J Gerontol A Biol Sci Med Sci 65: 792-799, 2010. 
32. Woo J, Yu R, Tang $\mathrm{N}$ and Leung J: Telomere length is associated with decline in grip strength in older persons aged 65 years and over. Age (Dordr) 36: 9711, 2014.

33. Marzetti E, Lorenzi M, Antocicco M, Bonassi S, Celi M, Mastropaolo S, Settanni S, Valdiglesias V, Landi F, Bernabei R and Onder G: Shorter telomeres in peripheral blood mononuclear cells from older persons with sarcopenia: Results from an exploratory study. Front Aging Neurosci 6: 233, 2014.

34. Singh A, Kukreti R, Saso L and Kukreti S: Oxidative stress: Role and response of short guanine tracts at genomic locations. Int J Mol Sci 20: 4258, 2019.

35. Fouquerel E, Lormand J, Bose A, Lee HT, Kim GS, Li J, Sobol R, Freudenthal BD, Myong S and Opresko PL: Oxidative guanine base damage regulates human telomerase activity. Nat Struct Mol Biol 23: 1092-1100, 2016.
36. Brandl A, Hartmann A, Bechmann V, Graf B, Nerlich M and Angele P: Oxidative stress induces senescence in chondrocytes. J Orthop Res 29: 1114-1120, 2011.

37. Yudoh K, van Nguyen T, Nakamura H, Hongo-Masuko K, Kato $\mathrm{T}$ and Nishioka K: Potential involvement of oxidative stress in cartilage senescence and development of osteoarthritis: Oxidative stress induces chondrocyte telomere instability and downregulation of chondrocyte function. Arthritis Res Ther 7: R380-R391, 2005.

38. Martin JA, Brown TD, Heiner AD and Buckwalter JA: Chondrocyte senescence, joint loading and osteoarthritis. Clin Orthop Relat Res (427 Suppl): S96-S103, 2004.

39. Martin JA, Brown T, Heiner A and Buckwalter JA: Post-traumatic osteoarthritis: The role of accelerated chondrocyte senescence. Biorheology 41: 479-491, 2004. 